\title{
Dashboard digital para el monitoreo de indicadores y metas de los proyectos de consultores San Martín E.I.R.L.
}

\author{
Digital dashboard for the monitoring of indicators and goals \\ of consultores San Martín E.I.R.L.
}
Calle Paz, Ibelicet Ixamar ${ }^{1[0000-0002-6830-5754]}$ y Valles-Coral, Miguel Angel 1 [0000-0002-8806-2892]
${ }^{1}$ Universidad Nacional de San Martín,Tarapoto, Perú ibixcpazegmail.com

Resumen. Cada día más las empresas, ya sean pequeñas, medianas o grandes, necesitan el monitoreo de sus actividades, el desempeño de cada trabajador y como llevan sus finanzas. El uso de herramientas que lleven el monitoreo justamente de estos factores están siendo requeridos, una de ellas es el Dashboard Digital como tal, puesto que permite de manera táctica y operativa conocer el estado general de la empresa. Implantar un Dashboard Digital o Tablero de Control, implica que reconozca y establezca cuáles son los indicadores claves, analizar los factores en riesgo y tomar medidas y decisiones que permitan obtener resultados óptimos y asegurar el desarrollo. En esta investigación se plantea demostrar el uso del Dashboard Digital como herramienta para obtener resultados mediante el monitoreo, ayude a tomar decisiones estratégicas, se identifique los riesgos mientras a la par existan mejoras para la productividad, siendo estos pilares importantes para garantizar la competitividad de una empresa. La puesta en marcha de esta solución fue una meta muy significativa para la empresa puesto que otorgó una visión mucho más amplia acerca de la labor que se lleva a cabo y del servicio que se otorga. Esto permitió ver resultados mucho más concretos y analizables permitiendo a la gerencia tomar decisiones precisas en momentos oportunos. Finalmente, se sugiere que las empresas adopten estas herramientas, puesto que no solo obtendrán beneficios financieros sino también beneficios competitivos y estratégicos que marcaran la calidad de sus servicios y se posicionaran en un escalón más hacia el camino del éxito empresarial.

Palabras Claves: dashboard digital, indicadores, monitoreo, tablero de control, toma de decisiones

Citar como: Calle Paz, I. I., \& Valles Coral, M. A. (2021). Dashboard digital para el monitoreo de indicadores y metas de los proyectos de consultores San Martín E.I.R.L. Revista Científica De Sistemas E Informática, 1(1), 24-36. https://doi.org/10.51252/rcs i.v1i1.94

Recibido: $15 / 11 / 2020$

Revisado: $15 / 12 / 2020$

Publicado: 31/01/2021
Abstract. Every day more companies, whether small, medium or large, need to monitor their activities, the performance of each worker and how they carry their finances. The use of tools that take the monitoring of just these factors are being required, one of them is the Digital Dashboard as such, since it allows tactically and operationally to know the general state of the company. Implement a Digital Dashboard or Control Board, implies that it recognizes and establishes which are the key indicators, analyze the factors at risk and take measures and decisions that allow to obtain optimal results and ensure the development. This research aims to demonstrate the use of the Digital Dashboard as a tool to obtain results through monitoring, help to make strategic decisions, identify risks while at the same time there are improvements for productivity, these pillars being important to guarantee the competitiveness of a company. The implementation of this solution was a very significant goal for the company since it gave a much broader vision about the work that is carried out and the service that is granted. This allowed us to see much more concrete and analyzable results, allowing management to make precise decisions at opportune moments. Finally, it is suggested that companies adopt these tools, since they will not only obtain financial benefits but also competitive and strategic benefits that will mark the quality of their services and will position themselves one step further towards the path of business success.

Keywords: control board, decision making, digital dashboard, indicators, monitoring 


\section{Introducción}

La presente investigación titulada Dashboard Digital para el monitoreo de indicadores y metas de la empresa Consultores San Martin E.I.R.L., fue planteada según la siguiente hipótesis ¿Con el uso de un Dashboard Digital se mejorará el monitoreo de los indicadores y metas de los proyectos de Consultores San Martín?, puesto que la empresa al contar con formatos donde se guardan los datos mas no la información, éstos solo son utilizados para uso referencial, mas no como uso informativo y que ayude en la toma de decisiones., lo que se requiere es hacer uso de las herramientas de tecnologías de la información que hoy en día son tomadas con mucho más valor en la gerencia de una empresa justamente para tomar mejores decisiones y llevar un mejor control de los recursos que poseen, por tal motivo se propuso contar con el uso de un Dashboard Digital. Lo que se pretende es dar solución a la problemática presente en la empresa Consultores San Martín que viene acarreando desde muchos años atrás, que consta del deficiente monitoreo con respecto a la elaboración de los proyectos, estos no son entregados a tiempo por tener pendiente proyectos atrasados; el desempeño de los trabajadores, si la labor ejercida es la adecuada, si la atención brindada es efectiva; los pagos que en muchas ocasiones son obviados por no registrar las fechas y los montos de cada proyecto, gastos que genera cada proyecto (conocer si ex ${ }^{*}$ : perdida o monto ganado).

La investigación presenta tres capítulos los cuales se detallan a continuación: En el primer capítulo "Revisión Bibliográfica" se detallan seis antecedentes de investigación, el marco teórico que esclarece puntos importantes relacionados con el tema a desarrollar como son definición de términos: monitoreo, indicadores, tipología de indicadores, definición de Dashboard. En el segundo capítulo "Material y Métodos" donde se especifica el universo y la muestra que son una variada cantidad de proyectos. El tipo de diseño de investigación es Descriptivo - Comparativo, la cual se basa en la medición y comparación de la variable respuesta antes y después de la exposición del sujeto a la intervención experimental. Cuadros de excel se usaron en un inicio como instrumentos de medición, las entrevistas y las encuestas fueron necesarias para el levantamiento de información y para la obtención de resultados después de implantada la herramienta. En el tercer capítulo "Resultados y Discusión”, se toma en consideración la prueba de la hipótesis : "Con el uso del Dashboard Digital se mejorará el monitoreo de los indicadores y metas de los proyectos de Consultores San Martín E.I.R.L.", se realizará la comprobación de ésta mediante un estudio estadístico con los datos obtenidos mediante el uso de la herramienta puesta en estudio, además mostramos los resultados obtenidos mediante el uso de reportes y gráficos los cuales nos permiten tener una mejor forma de ver el manejo de la problemática y que ésta se solucione y pueda brindar conocimiento táctico para una mejor toma de decisiones empresariales. En los resultados también se mencionan aquellos resultados obtenidos de acuerdo a los objetivos

Citar como: Calle Paz, I. I., \& Valles Coral, M. A. (2021). Dashboard digital para el monitoreo de indicadores y metas de los proyectos de consultores San Martín E.I.R.L. Revista Científica De Sistemas E Informática, 1(1), 24-36. https://doi.org/10.51252/rcsi.v1i1.94 
específicos plasmados en el estudio de investigación los cuales son: Desarrollar un Dashboard Digital; Mejorar el monitoreo de indicadores y metas de los proyectos; Evaluar el impacto del uso del Dashboard sobre el monitoreo de los indicadores y metas de los proyectos de la empresa Consultores San Martín E.I.R.L., Y finalmente la discusión de los resultados que se basan justamente al estudio previo de los antecedentes de las investigaciones.

Como últimos puntos tenemos a las conclusiones y recomendaciones; en las conclusiones se pudo esclarecer que oportunamente se pudo brindar una solución a la empresa, logrando de esta manera que la herramienta Dashboard Digital sea óptima para estos casos, además se puede concluir también que se tuvo un gran impacto positivo con el uso de la metodología para construcción de Dashboard. Las recomendaciones pretenden ser una fuente o guía de trabajo metodológico para la empresa como así también para los lectores de este trabajo de investigación.

\section{Revisión Bibliográfica}

Terán Tabango, (2015) En el trabajo de titulación "Dashboard de Ventas y Módulo de Reporteo Web para la empresa Pinto S.A. ubicada en la ciudad de Quito" tiene como conclusión lo siguiente: La solución del "Dashboard de Ventas y Módulo de Reporteo Web para la Empresa Pinto S.A ubicada en la ciudad de Quito”, permite a las áreas de la empresa independizarse del departamento de sistemas, utilizando una interfaz de aplicación fácil de usar en diferentes sitios o áreas de trabajo, el usuario utiliza los servicios de: reporteo y Dashboard con el fin de generar información que les sea de utilidad a los todos los usuarios del sistema.

Tejada Dussán, (2015) en el proyecto de investigación "Implementación Tablero de control de ventas POS para Nestle de Colombia", concluyen que: El tablero de control será una buena solución para facilitar la gestión de los directores, al proveer una herramienta de conocimiento del negocio, de fácil entendimiento, que muestre de una forma resumida la labor que está desempeñando su fuerza de ventas y que a su vez logre centrar sus esfuerzos en cumplir sus funciones básicas, objetivos estratégicos y encaminarse a alcanzar su visión, sin tener que hacer esfuerzos innecesarios orientados a dispendiosos controles de información. Con la implementación del tablero de control de las ventas POS, se va a tener un manejo más rápido, eficiente y oportuno de la información, haciendo posible que las estrategias de negocio y las mejoras continuas puedan ser aplicadas dentro de los tiempos de operación, lo que trae beneficios para dar cumplimiento con su misión, dar los resultados esperados y que se cumplan las metas que se planifican cada mes.

Mena Vargas, (2017) en el proyecto de titulación "Desarrollo e Implementación del módulo de admisión y medicina general con reportería y tablero de control de citas para el sistema medico integrado del policlínico Dra. Jenny Ayoví Corozo de la ciudad de Guayaquil” concluye que: La

Citar como: Calle Paz, I. I., \& Valles Coral, M. A. (2021). Dashboard digital para el monitoreo de indicadores y metas de los proyectos de consultores San Martín E.I.R.L. Revista Científica De Sistemas $E$ Informática, 1(1), 24-36. https://doi.org/10.51252/rcsi.v1i1.94 
visualización de un tablero de control de citas en el cual se indique la especialidad, doctor, fecha/hora y estado, podrá ayudar a la gestión de las citas médicas activas para el día en curso. Para desarrollar la solución de inteligencia de negocios propuesta, se analizó dos metodologías reconocidas como son Hefesto y Kimball.

Aucaylle Nieto \& Altamirano Condori, (2017) en el proyecto de tesis "Impacto del uso de un tablero de control de desempeño en seguridad y salud en el trabajo para empresas contratistas, en el proyecto "Mejoras a la seguridad energética del país y desarrollo del gasoducto Sur Peruano", concluye con lo siguiente: El uso del tablero de control para empresas contratistas impacta positivamente en los indicadores de Gestión de Seguridad y Salud en el trabajo, en el proyecto "Mejoras a la Seguridad Energética del País y Desarrollo del Gasoducto Sur Peruano, 2016".

Jara Muñoz, (2015) en la tesis "Implementación de Tableros de Control SAP BO para los indicadores de operación de la división minería y energía”, tiene como conclusión lo siguiente: La alta disponibilidad de la información de Minería y Energía a través de la implementación de tableros de control SAP BO garantiza un servicio disponible en todo momento, permitiendo que el personal propio de la división y sus clientes puedan realizar el análisis respectivo y tomar decisiones óptimas en sus procesos de negocio.

Jara Rodriguez, (2017) en la tesina "Implementación de tablero de control de gestión para toma de decisiones en una empresa de servicios generales" El tablero de control ubica los procesos de la organización, de modo tal, que cada uno asume su importancia real y su peso específico en el contexto de la organización, racionalizando la relación entre las áreas con base en su trascendencia para el cumplimiento de la misión y el logro de la visión.

\section{Materiales y Métodos}

Para este proyecto de investigación se realizó un estudio de Diseño Descriptivo - Comparativo en la cual se basa en la medición y comparación de la variable respuesta antes y después de la exposición del sujeto a la intervención experimental.

Los pasos a continuación presentados son parte de la metodología a utilizar.

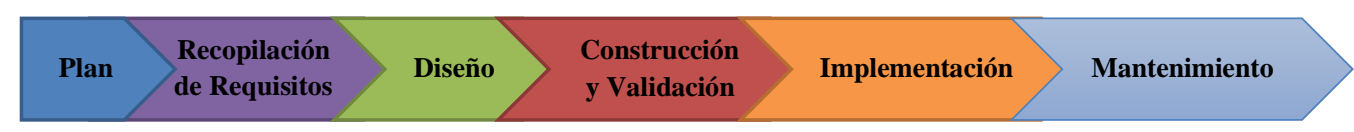

Figura 1: Metodología para Creación del Dashboard

\section{Plan, Recopilación de requisitos, diseño, y Construcción y Validación}

El presente proyecto se inició con el primer punto llamado levantamiento de información, en este punto se obtuvo información detallada del ciclo de vida que tiene un proyecto, desde la 
contratación del servicio que brinda la empresa, así como el resultado que provee a los usuarios que recurren a ella para obtener resultados y elaborar un estudio final.

Como segundo punto se hace referencia a la identificación de requisitos mediante las reuniones que se tuvo con los representantes de la empresa Consultores San Martín, en pocas palabras con el Gerente y el Asistente de Gerencia, para demostrar a detalle cómo se encuentra la empresa con respecto a la problemática de monitoreo de los procesos que contiene cada proyecto que se realiza, desde inicio, ejecución, culminación hasta la entrega de informes finales. En este punto también se llegó a identificar los indicadores, cuya identificación en la ejecución del proyecto permitirá lograr objetivos cualitativos y cuantitativos para la empresa, los Indicadores a monitorear están basados en el tiempo (¿Cuánto tiempo conlleva realizar los proyectos?), eficiencia y eficacia del trabajador (indicadores de actividad) e indicadores de estructura financiera (¿Cuánto es la perdida financiera por aplazar un día más la elaboración de un proyecto?); en lo referente a las metas es que cada proyecto a realizarse en la empresa sea entregado en la fecha indicada y cumpliendo con las expectativas del cliente. Teniendo a disposición la información recopilada en las entrevistas y reuniones con el gerente, el asistente de gerencia, trabajadores y usuarios (clientes) se prosiguió con la identificación de los datos más distinguidos que nos permitan y ayuden a llevar a cabo el monitoreo en el proceso de construcción de los proyectos solicitados a la empresa.

El siguiente paso fue la creación del Dashboard Digital de acuerdo a los indicadores que han sido identificados y de acuerdo al sistema transaccional elaborado para el ingreso de datos. Estos indicadores son representados mediante gráficos semicirculares y gráficos de barras; esto con el fin de demostrar información concisa e importante al gerente de la empresa y este pueda tomar decisiones o autoevaluar las situaciones en riesgo o con aquellas que puedan variar el buen funcionamiento de la empresa.

Citar como: Calle Paz, I. I., \& Valles Coral, M. A. (2021). Dashboard digital para el monitoreo de indicadores y metas de los proyectos de consultores San Martín E.I.R.L. Revista Científica De Sistemas E Informática, 1(1), 24-36. https://doi.org/10.51252/rcsi.v1i1.94 


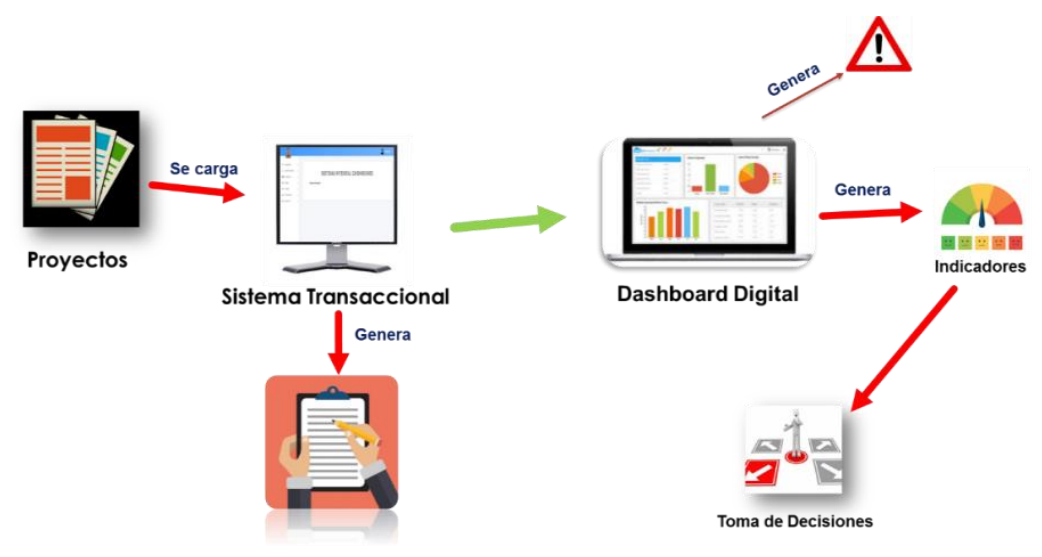

Figura 2: Propuesta final

\section{a) Implementación de Front End}

Los gráficos que se utilizaron para el desarrollo del Dashboard fueron:

Gráficos en Barras: El gráfico de barras implementado en el Dashboard y que se muestran en el mismo permiten ver el avance (porcentaje) de las actividades que conlleva cada proyecto, además dará a conocer el pago y el gasto puesto esto en soles.

Gráficos Gauge: El gráfico gauge implementado en el Dashboard ver el porcentaje de los proyectos, de los pagos y de los gastos. Con respecto a los proyectos y los pagos; el color rojo indica que existen retrasos tanto en el desarrollo de actividades y en las cuotas que tienen que pagar los clientes, el color amarillo indica que se avanzó más del 50\% y el color verde indica que se realizó completamente al $100 \%$. Con respecto a los gastos funciona de manera diferente; el color verde significa que no se realizó muchos gastos, el color amarillo indica que se está gastando más del 50\% de lo que se ha cobrado por desarrollar el proyecto y el indicativo rojo significa que se gastó la totalidad del proyecto sin generar ganancias.

Citar como: Calle Paz, I. I., \& Valles Coral, M. A. (2021). Dashboard digital para el monitoreo de indicadores y metas de los proyectos de consultores San Martín E.I.R.L. Revista Científica De Sistemas E Informática, 1(1), 24-36. https://doi.org/10.51252/rcsi.v1i1.94 


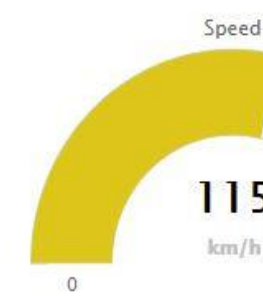

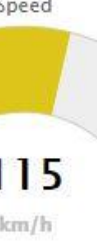

\section{5}

ith

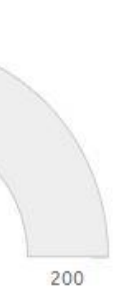

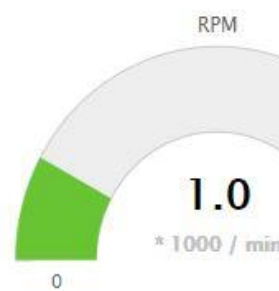

1.0

Figura 3: Gráfico Gauge utilizado en el Dashboard Digital

Sistema Alertas: Las Alertas se encuentran al iniciar el sistema, esto de una forma de notificación, en el cual se muestran proyectos que existen actividades pendientes o se encuentran vencidas y necesitan su pronta observación, de igual manera se muestran notificaciones de los pagos.

\section{b) Implementación de Consultas}

Se llevó a cabo la elaboración de consultas en la base de datos, con referencia a los gastos, se combinan los proyectos y pagos para conocer que gastos se han generado. Los proyectos y los pagos son necesarios en esta consulta para conocer de qué proyecto y de que pago se están realizando gastos.

\section{c) Configurar programación, actualización y seguridad}

En el sistema Dashboard se han generado perfiles, el perfil de Gerente se encarga de los módulos de los pagos, gastos, indicadores y reportes. Al observar los reportes gráficos permite tomar las medidas necesarias para mejorar el desarrollo de las actividades que genera desarrollar un proyecto como son: el cumplimiento de plazos de presentación de informes finales, al mismo tiempo esto permite demostrar que trabajador está desarrollando sus actividades correspondientes, así como también nos indica que proyectos faltan cancelar, están cancelados y que gastos se pueden realizar indicando de que proyecto.

\section{Implementación (Deploy)}

Para llevar a cabo el proceso de implementación, se procedió a la instalación del Dashboard. La instalación del Dashboard se hizo dentro del panel del servidor de Amazon (AWS).

\section{Mantenimiento (Maintain)}

Una vez implantado el sistema en la plataforma de AWS y otorgado una dirección web, el mantenimiento se realizó periódicamente. Se llevó a cabo la capacitación al personal de la empresa sobre el uso del Dashboard.

Citar como: Calle Paz, I. I., \& Valles Coral, M. A. (2021). Dashboard digital para el monitoreo de indicadores y metas de los proyectos de consultores San Martín E.I.R.L. Revista Científica De Sistemas E Informática, 1(1), 24-36. https://doi.org/10.51252/rcsi.v1i1.94 


\section{Resultados y Discusión}

Este proyecto de investigación tuvo como propósito principal mejorar el monitoreo de los indicadores y metas de los proyectos de Consultores San Martín, puesto que la empresa al contar con importante flujo de información de los proyectos que brinda; obliga a la misma a adoptar modelos de gestión que le permitan hacer un óptimo control y monitoreo de indicadores y metas que poseen los proyectos, esto contribuirá a la mejor toma de decisiones y del mismo modo al crecimiento de la empresa. La herramienta empleada para el análisis estadístico de la investigación fue "T - Student".

\section{Prueba de T-Student}

Este tipo de prueba es ideal cuando se desea comparar las medidas de dos grupos que tienen una distribución normal con número de observaciones menores a 30 y se usa el estimador $\mathrm{s}^{2}$.

Según la prueba de muestras independientes los resultados nos indican que el T-calculado $\left(\mathrm{T}_{\mathrm{c}}\right)$ tiene valor de -14.947

Este valor lo contrastaremos con el T-tabla (Tt); Se busca en la tabla de T-Student con 2(n-1) grados de libertad o sea 38 y se encuentra que el valor tabular es de -1.686 al $95 \%$ de probabilidad $(\mathrm{Tc}<\mathrm{Tt})$

Se trabajó con una cola unilateral izquierda, puesto que $\mathrm{H} 1 ; \mu_{\mathrm{a}}<\mu_{\mathrm{d}}$

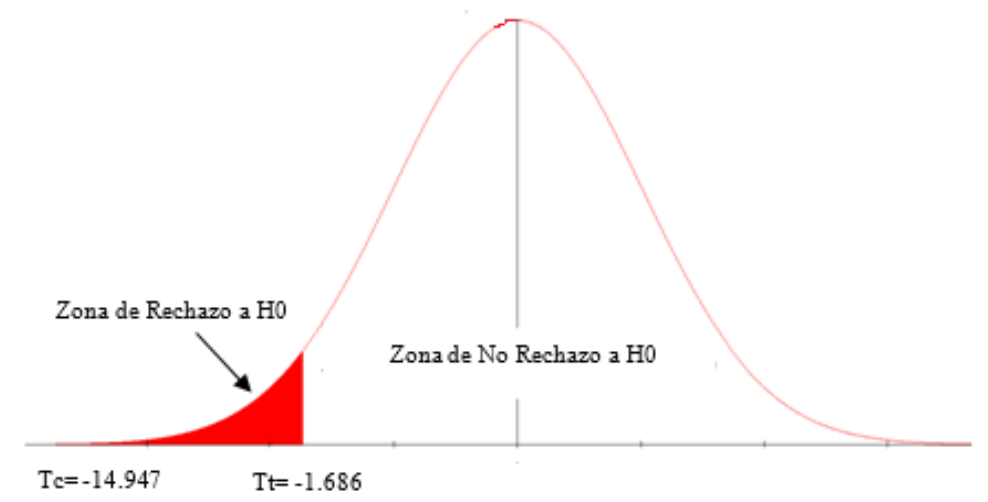

Figura 4: Distribución T-Student

Decisión:

$\mathrm{Si}(\mathrm{Tc}<\mathrm{Tt})-14.947<-1.686$ rechazamos la hipótesis nula.

Como $\mathrm{Tc}=-14.947$ es mayor que $\mathrm{Tt}=-1.686$, entonces rechazamos la H0 (Hipótesis Nula) y por consiguiente aceptamos la Hipótesis alternativa, de esta manera se puede afirmar que: Con el uso

Citar como: Calle Paz, I. I., \& Valles Coral, M. A. (2021). Dashboard digital para el monitoreo de indicadores y metas de los proyectos de consultores San Martín E.I.R.L. Revista Científica De Sistemas $E$ Informática, 1(1), 24-36. https://doi.org/10.51252/rcsi.v1i1.94 
del Dashboard Digital si se mejorará el monitoreo de indicadores y metas de los proyectos de Consultores San Martín E.I.R.L.

Una vez analizados los requerimientos obtenidos y después de levantar la información se logró crear un Sistema Integral que permitirá que la herramienta (Dashboard Digital) funcione correctamente. Usando la Metodología para el Diseño de Dashboard de (Robalino Martínez, 2017), se ha construido el Dashboard Digital, teniendo como principal interesado al Gerente General y a la Asistente de Gerencia.

A continuación se muestran el resultado de la medición, comparación y aplicación del instrumento, a los indicadores de la investigación a fin de demostrar la validez de la hipótesis planteada. Se aplicó una encuesta pre-test y post-test para conocer que opinan los clientes acerca del monitoreo de indicadores y del servicio que brinda la empresa. Se distinguió tres dimensiones en el instrumento de investigación, los cuales son: Calidad del Sistema, Evaluación y Satisfacción; los resultados a continuación están presentados mediante resultados pre y post del instrumento de evaluación.

Tabla 1
\begin{tabular}{ccc} 
Opinión sobre el impacto del Dashboard sobre el monitoreo de los indicadores y metas de los proyectos. \\
\cline { 2 - 3 } Opinión & \multicolumn{2}{c}{ Dashboard Digital } \\
\cline { 2 - 3 } & Pre & Post \\
Malo & $55 \%$ & $35 \%$ \\
Regular & $45 \%$ & $65 \%$ \\
Bueno & $0 \%$ & \\
Fuente: Datos obtenidos de la encuesta a clientes de la empresa son respecto a las tres dimensiones
\end{tabular}

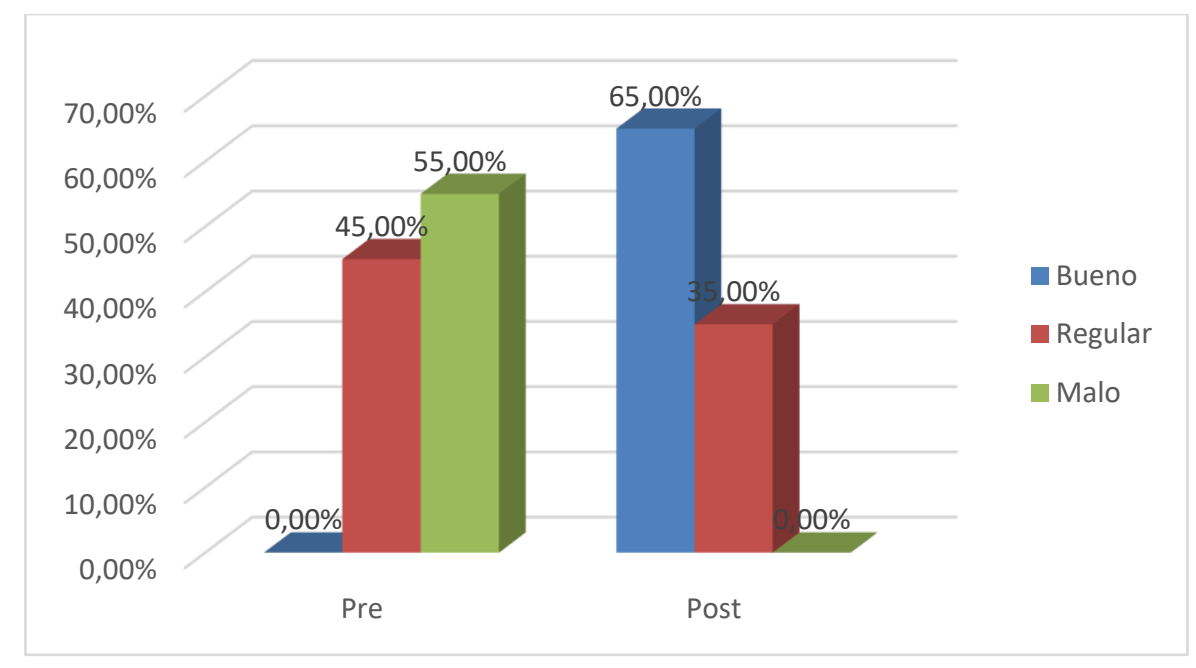

Figura 5: Opinión sobre el Monitoreo de Indicadores y Metas de los proyectos 


\section{Interpretación y discusión}

Según el resultado que se tiene en la tabla 1 y figura 5, se detalla y evidencia el resultado del pre y post análisis, se explicarán los resultados post, de la cual se evidencia que el $0 \%$ de los clientes opinan que el impacto será Malo, un 35\% opinan regular y el 65\% opinan que será Bueno.

El monitoreo de indicadores y metas de los proyectos, mediante un instrumento como en este caso es el Dashboard Digital, permite que los resultados de nuestros análisis se conviertan en factores determinantes para el buen desempeño de la empresa, tal y como lo aclara Tejada Dussán, (2015), para una compañía es muy valioso contar con instrumento que contenga los indicadores de desempeño, con la cual surjan elementos de diagnóstico y así se puedan orientar las decisiones gerenciales. Este tablero va a ser una herramienta que va a proporcionar un control de la gestión del negocio, control en la gestión que la fuerza de ventas de la compañía está realizando y este se va a convertir en un facilitador de toma de decisiones. Nuestra propuesta coincide con los resultados propuestos por Tejada Dussán, (2015), con la implementación del tablero de control de las ventas POS, se va a tener un manejo más rápido, eficiente y oportuno de la información, haciendo posible que las estrategias de negocio y las mejoras continuas puedan ser aplicadas dentro de los tiempos de operación, lo que trae beneficios para dar cumplimiento con su misión, dar los resultados esperados y que se cumplan las metas que se planifican cada mes; en la que se concluye que ambas propuestas tienen como finalidad tener un manejo de la información de manera más oportuna, rápida y eficiente, puesto que en nuestro caso el manejo de la información es de forma diaria y con mucho más precisión. La visualización constante de las actividades, de la generación de pagos y gastos y el detalle de estos, permite que la empresa gestione adecuadamente su información, de igual forma e importancia argumenta Mena Vargas, (2017), sobre el visualizar un Dashboard o tablero de Control, "La visualización de un tablero de control de citas en el cual se indique la especialidad, doctor, fecha/hora y estado, podrá ayudar a la gestión de las citas médicas activas para el día en curso”.

Citar como: Calle Paz, I. I., \& Valles Coral, M. A. (2021). Dashboard digital para el monitoreo de indicadores y metas de los proyectos de consultores San Martín E.I.R.L. Revista Científica De Sistemas $E$ Informática, 1(1), 24-36. https://doi.org/10.51252/rcsi.v1i1.94 


\section{Conclusiones}

En primer lugar el proyecto de investigación ha permitido desarrollar un tablero de control para el monitoreo de los indicadores y metas de los proyectos de consultores San Martín, el cual mediante su uso marco un punto importante entre la situación actual y la de antes, además permitió gestionar de una forma más ordenada las actividades, los pagos y gastos que en cierta forma es la funcionalidad operativa y táctica del manejo de la información, proporcionando indicadores y metas, haciendo que el cumplimiento de estas ayuden a mejorar los resultados cuantitativos y cualitativos de la empresa, así como también ayuden a tomar mejores decisiones gerenciales. En segundo lugar, mediante el desarrollo de un Dashboard Digital las mejoras del monitoreo de indicadores y metas de los proyectos son palpables, porque los proyectos son entregados a tiempo, los trabajadores realizan las actividades de acuerdo al cronograma, así como también los pagos y los gastos son monitoreados de igual manera a los cronogramas y a las alertas diarias; esto comprobado gracias al instrumento utilizado como es la encuesta. Además se pudo verificar y aceptar la hipótesis alterna del estudio que con el uso de un Dashboard digital se mejorará el monitoreo de indicadores y metas de los proyectos, por haberse conseguido mediante análisis estadístico resultados significativos para la investigación. Finalmente, mediante el Dashboard Digital se ha podido evaluar el impacto que esta fomenta sobre el monitoreo de indicadores y metas de la empresa Consultores San Martín, así como también las mejoras que permiten su uso, se concluye además que mediante el análisis estadístico realizado, al instrumento de investigación aplicado, el estado de malo - regular a regular - bueno será de un 55\% - 45\% a 35\% - 65\%, se ven reflejados en la Tabla 12 y figura 35, además mediante la prueba de la hipótesis alterna, se puede mencionar que la creación de un Dashboard resulto mucho más favorable para el ambiente gerencial de la empresa.

\section{Agradecimientos}

A la gerencia de la empresa Consultores San Martín, quienes me brindaron su confianza y permitieron que este proyecto se desarrolle y ejecute.

Un agradecimiento especial al Instituto de Investigación y Desarrollo de la UNSM-T, que a través del Concurso de Proyectos de Investigación para Tesis a Nivel de Pregrado 2018, y a su plana directiva se hizo posible la ejecución de este trabajo de investigación.

Citar como: Calle Paz, I. I., \& Valles Coral, M. A. (2021). Dashboard digital para el monitoreo de indicadores y metas de los proyectos de consultores San Martín E.I.R.L. Revista Científica De Sistemas $E$ Informática, 1(1), 24-36. https://doi.org/10.51252/rcsi.v1i1.94 


\section{Referencias bibliográficas}

Arriagada, R. (2002). Diseño de un sistema de medición de desempeño para evaluar la gestión municipal: una propuesta metodológica. Santiago de Chile.

Aucaylle Nieto, J. R., \& Altamirano Condori, G. (2017). Impacto del uso de un tablero de control de desempeño en seguridad y salud en el trabajo para empresas contratistas, en el proyecto \&quot;mejoras a la seguridad energética del país y desarrollo del gasoducto sur peruano\&quot;, 2016. Universidad Andina del Cusco. Universidad Andina del Cusco.

Banco Interamericano De Desarrollo. (1997). Evaluación: Una herramienta de gestión para mejorar el desempeño de los proyectos.

Baumgarten, J. P. (2016). Metas: secretos y tčnicas para alcanzar tus suegs y metas. Babelcube Inc.

Berumen Milburn, J. (2010). Monitoreo y Evaluación de proyectos. Escuela Latinoamericana de Cooperación y Desarrollo.

Consejo NAcional de Evaluación de la Política de Desarrollo Social. (2014). Manual para el diseño y la construcción de indicadores. Instrumentos principales para el monitoreo de programas sociales de México. Mexico.

Delaux, H. S. (2017). Planeamiento Estrategico y Creación de Valor: Reestructurar en Crisis. Argentina: BoD - Book on Demand.

FAO. (2005). Informatización de cooperativas agrícolas: guía práctica.

Fleitman, J. (2007). Evaluación integral para implantar modelos de calidad. Pax México.

Garcia, V. (2013). Fase Monitoreo-y-Evaluacion-de-proyectos.

Gens, F. (2004). rioridades empresariales para la Hoja de ruta dinámica de TI. Framingham: DC.

Hernández Becerra, E., \& Food and Agriculture Organization of the United Nations. (1993). Monitoreo y evaluatión de logros en proyectos de ordenación de cuencas hidrográficas. FAO.

Jara Muñoz, C. E. (2015). Implementación de Tableros de control SAP BO para los indicadores de operación de la división minería y energía. Universidad Nacional San Ignacio de Loyola.

Jara Rodriguez, K. L. (2017). Implementación de tablero de control de gestión para la toma de decisiones en una empresa de servicios generales. Universidad Nacional Mayor de San Marcos.

Luecke, R., Hall, B. J., \& San Millán, E. G. (2007). Gestión del desempeño : evalúe y mejore la eficacia de sus colaboradores. Eds. Deusto.

Lusthaus, C., Adrien, M.-H., Anderson, G., \& Carden, F. (2001a). MEJORANDO EL

Citar como: Calle Paz, I. I., \& Valles Coral, M. A. (2021). Dashboard digital para el monitoreo de indicadores y metas de los proyectos de consultores San Martín E.I.R.L. Revista Científica De Sistemas E Informática, 1(1), 24-36. https://doi.org/10.51252/rcsi.v1i1.94 
DESEMPEÑO DE LAS ORGANIZACIONES Método de autoevaluación. Retrieved from http://www.idrc.ca/es/ev-9370-201-1-DO_TOPIC.html

Lusthaus, C., Adrien, M.-H., Anderson, G., \& Carden, F. (2001b). MEJORANDO EL DESEMPEÑO DE LAS ORGANIZACIONES Método de autoevaluación.

Membrado Martínez, J. (2013). METODOLOGÍAS AVANZADAS PARA LA PLANIFICACIÓN Y MEJORA. Ediciones Diaz de Santos.

Mena Vargas, E. P. (2017). DESARROLLO E IMPLEMENTACIÓN DEL MÓDULO DE ADMISIÓN Y MEDICINA GENERAL CON REPORTERÍA Y TABLERO DE CONTROL DE CITAS PARA EL SISTEMA MÉDICO INTEGRADO DEL POLICLÍNICO DRA. JENNY AYOVÍ COROZO DE LA CIUDAD DE GUAYAQUIL. Guayaquil.

Minitab. (n.d.). ¿Qué son los grados de libertad?

Robalino Martínez, A. D. (2017). Metodología para el diseño de Dashboards orientado hacia el registro de evidencias en el proceso de evaluaciones institucionales. Universidad Internacional de La Rioja.

Tejada Dussán, O. F. (2015). IMPLEMENTACIÓNTABLERO DE CONTROL DE VENTAS POS PARA NESTLE DE COLOMBIA IMPLEMENTATION DASHBOARD OF POS SALES FOR NESTLE OF COLOMBIA - PDF.

Terán Tabango, M. V. (2015). Dashboard de Ventas y Módulo de Reporteo Web para la empresa Pinto S.A ubicada en la ciudad de Quito. Universidad Regional Autónoma de los Andes.

Valle, Otto y Rivera, O. (2008). Monitoreo e indicadores. Guatemala: Organizacion de Estados Iberoamericanos. 